\title{
Late presentation of paediatric patients to clinics: a major barrier to uptake of laboratory services in nigeria
}

co Okolo

\section{From 3rd International Conference on Prevention and Infection Control (ICPIC 2015)}

Geneva, Switzerland. 16-19 June 2015

\section{Introduction}

In Nigeria, paediatric patients present to health clinics at critical states that leave the medical doctors with no other choice but to blindly prescribe drugs without proper diagnostic tests.

\section{Objectives}

This study was aimed at determining effect of latepresentation of paediatric malaria cases to the hospital on uptake of laboratory services.

\section{Methods}

Conducted study at the Medical Centre, University of Nigeria, Enugu Campus. Reviewed medical records contents of fifty-six paediatric patients from January 22 to March 23, 2014. Records were taken during clinic days at the OPD section. Malaria parasite test showing 3+++ and PCV below $39 \%$ of the patients involved were used as a major basis for sampling. The following inclusion criteria were used: age bracket of 1-12years, late presentation, very weak with severe fever, malaria test of $3+++$ and PCV below 39\% while exclusion criteria included adults, early presenters and patients presenting symptoms not related to malaria. The following were the main instruments used; structured questionnaires, medical records and Micro-haematocrit centrifuge.

\section{Results}

$55 \%$ the patients admitted did not use laboratory services until their first line of anti-malaria therapy ended; their PCV reading was recorded as $39 \% .45 \%$ of the patients presented to the medical centre during the weekend when the Lab Scientist was not on duty; they recorded lower PCV reading of 38\% during the week day when the Lab scientist had resumed.

\section{Conclusion}

Late presentation which comes either in the form of:

- Patients presenting late to the clinic with severe malaria symptoms. This leads to anti-malaria therapy for the paediatric patients without the necessary diagnostic tests in a bid to save their lives, or

- Patients presenting to the medical centre during the weekend when the only Lab Scientist working at the medical centre is off-duty.

All these act as barriers to uptake of laboratory services.

\section{Disclosure of interest}

None declared.

Published: 16 June 2015

doi:10.1186/2047-2994-4-S1-P265

Cite this article as: Okolo: Late presentation of paediatric patients to clinics: a major barrier to uptake of laboratory services in nigeria. Antimicrobial Resistance and Infection Control 2015 4(Suppl 1):P265.

HIV/AIDS, John Snow Incorporated, Nigeria 